\title{
A new quality metric for dermatology: The "SPA" (sunscreen, protective gear, and avoidance) questionnaire to assess sun protection practices
}

\author{
Austin Hamp ${ }^{5}$, Allene S. Fonseca', Zar Y. Kotlarsic ${ }^{2}$, Teresa Lanasa ${ }^{4}$, Amanda Schultz ${ }^{4}$, \\ Robert P. Dellavalle ${ }^{2,3,4}$, Cory A. Dunnick ${ }^{3,4}$
}

${ }^{1}$ Division of Dermatology, Department of Medicine, University of Washington, Seattle, WA, USA, ${ }^{2}$ Colorado School of Public Health, Aurora, CO, USA, ${ }^{3}$ University of Colorado School of Medicine, Dept. of Dermatology, Anschutz Medical Campus, Aurora, CO, USA, ${ }^{4}$ Department of Veterans Affairs, Eastern Colorado Health Care System, Dermatology Service, Denver, CO, USA, ${ }^{5}$ Arizona College of Osteopathic Medicine, Midwestern University, Glendale, Arizona, USA

Corresponding author: Austin Hamp, OMSIII, E-mail: ahamp79@midwestern.edu

\begin{abstract}
ABSRACT
Background: Given the importance of counseling patients on UV radiation exposure, a Sunscreen, Protective Gear, and Avoidance (SPA) Questionnaire was developed to assess patients' sun exposure and help physicians provide tailored counseling to their patients regarding safe sun protection practices. Materials and methods: Six Denver Veterans Affairs (VA) dermatology providers who were trained in using the questionnaire randomly selected patients in the Denver VA Dermatology clinic to pilot-test the questionnaire. Demographic information and responses were collected using the SPA Questionnaire. Results: 43/61 (70.5\%) of patients answered "no" to at least one sun protection strategy. Of these 43 patients, 40 (93\%) were provided with tailored counseling. Five out of six providers indicated that the questionnaire encouraged conversations about sun protection for skin cancer prevention. Conclusion: The SPA questionnaire is a simple measure of UV exposure risk, which can help clinicians determine the sun-protective behavior of patients and initiate dialogue to increase patient knowledge of sun protection practices.
\end{abstract}

Key words: Quality metric; questionnaire; UV exposure measure; screening tool; tailored counseling

\section{INTRODUCTION}

An effort has been made in dermatology to improve quality of care through the use of quality measures [1]. These measures serve as a means of assessing the quality at which physicians provide care to their patients. We developed a novel quality measure with the Sunscreen, Protective Gear, and Avoidance (SPA) Questionnaire, with the primary goal of providing rapid in office screening for sun protective behavior. The SPA questionnaire may function as a quick in office screening tool for sunscreen protective behavior similar to a CAGE questionnaire for evaluating alcohol dependence or mini-mental status exam for assessing cognitive impairment. With providers having limited time during appointments, the advantage of using a shorter survey like the SPA Questionnaire lies in the shorter time it takes to administer. Furthermore, the SPA questionnaire helps providers ensure their patients receive tailored counseling based on their sun exposure. With tailored counseling, information is provided based on individual needs [2].

\section{MATERIALS AND METHODS}

In order to pilot the SPA Questionnaire as a screening tool for high-risk sun behaviors, six Denver Veterans Affairs (VA) dermatology providers randomly selected patients (with a history of skin cancer?) seen in the Denver VA Dermatology clinic. The providers were

\footnotetext{
How to cite this article: Hamp A, Fonseca AS, Kotlarsic ZY, Lanasa T, Schultz A, Dellavalle RP, Dunnick CA. A new quality metric for dermatology: The "SPA" (sunscreen, protective gear, and avoidance) questionnaire to assess sun protection practices. Our Dermatol Online. 2021;12(e):e74.

Submission: 30.03.2021; Acceptance: 26.07.2021

DOI: 10.7241 /ourd.2021e.74
} 
trained in using the questionnaire and provided a reference handout on "Protecting Yourself from Skin Exposure" [3]. Demographic information and responses were collected using the SPA Questionnaire (Fig. 1). Providers were interviewed for feedback after the data collection was completed.

\section{RESULTS}

We found that 43/61 (70.5\%) of patients answered "no" to at least one sun protection strategy. Of these 43 patients, 40 (93\%) were provided with tailored counseling. Two of the three patients who were not provided tailored counseling were older and had physical limitations that would minimize the benefits of counseling. One patient was not given counseling for unknown reasons. Five out of six providers indicated that the SPA metric encouraged targeted conversations about sun protection for skin cancer prevention.

\section{DISCUSSION}

The U.S. Preventive Services Task Force (USPSTF) currently recommends counseling young adults, adolescents, children, and parents of young children about minimizing exposure to ultraviolet (UV) radiation for persons ages 6 months to 24 years with fair skin types to reduce their risk of skin cancer [4]. Furthermore, skin cancer prevention counseling is especially relevant for patients with a history of skin cancer. The SPA Questionnaire can be a very useful and convenient tool that both primary care physicians and dermatologists can utilize to screen their patients to determine which behaviors are high risk. Secondary benefits of administering

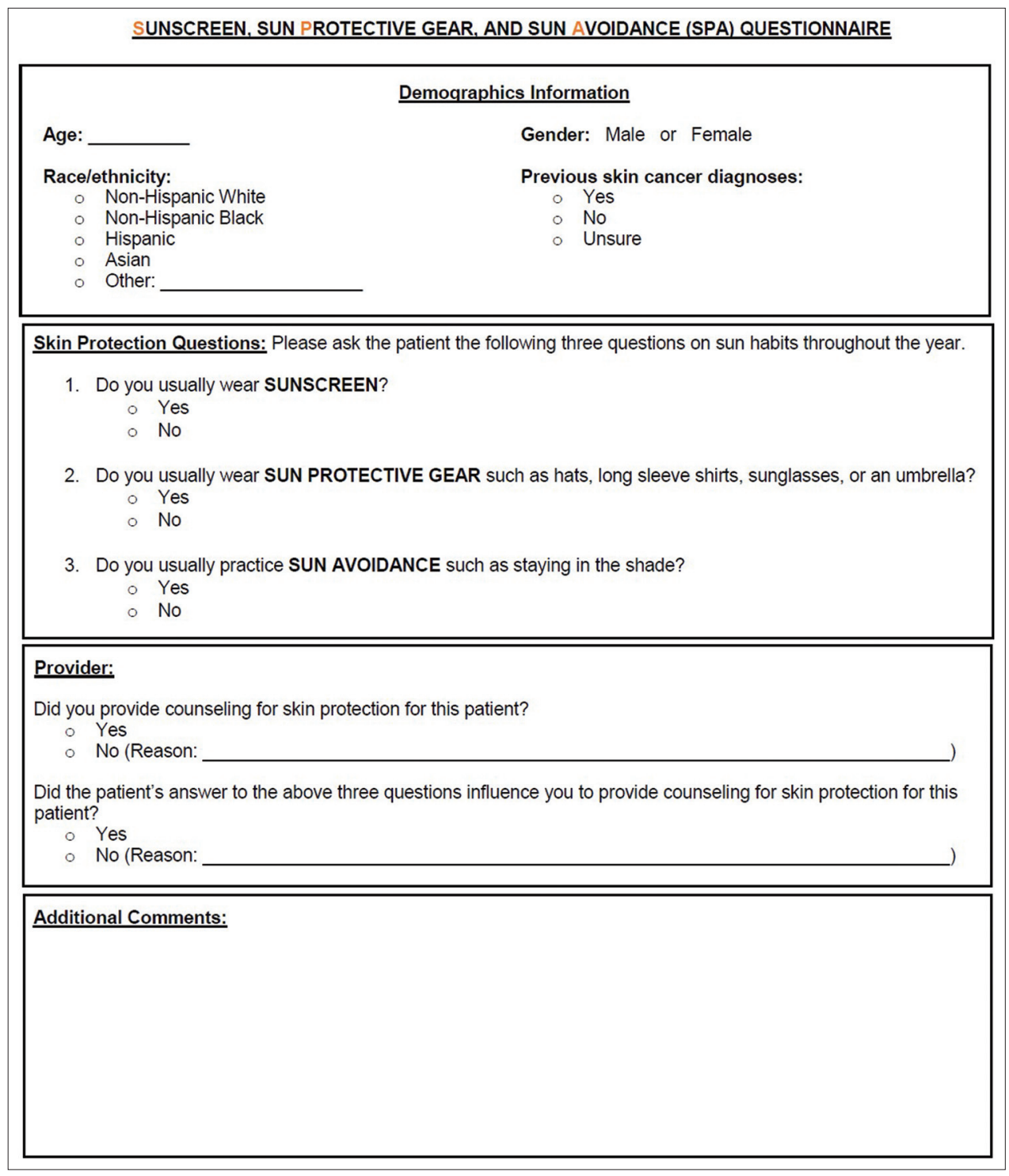

Figure 1: Sunscreen, Protective Gear, and Avoidance (SPA) questionnaire. 
the SPA Questionnaire include the short time it takes to administer, allowing physicians more time with the patient. Longer consultation time has been associated with better health outcomes [5]. Thus, with the short SPA Questionnaire, physicians will have more time for consultation, possibly leading to better health outcomes.

\section{CONCLUSION}

In conclusion, the SPA questionnaire is a simple measure of risky UV exposure behaviors. Posing these questions to patients may initiate dialogue and increase the knowledge of sun protection practices. It may be helpful to ask the questions in reverse order: assess sun avoidance, protective gear, and finally, sunscreen to emphasize that sunscreen is a secondary sun protection measure and sun avoidance primary. Further studies are needed to correlate sun exposure with the SPA questionnaire and to assess the effectiveness of tailored counseling to reduce UV exposure. Additional studies are needed to validate the SPA assay as a useful measure of quality in a diverse range of dermatology practices.

\section{Statement of Human and Animal Rights}

All the procedures followed were in accordance with the ethical standards of the responsible committee on human experimentation (institutional and national) and with the 2008 revision of the Declaration of Helsinki of 1975.

\section{Statement of Informed Consent}

Informed consent for participation in this study was obtained from all patients.

\section{REFERENCES}

1. Wilson RL, Feldman SR. Physician performance measures in dermatology. J Am Acad Dermatol. 2010;63:e29-35.

2. Hawkins RP, Kreuter M, Resnicow K, Fishbein M, Dijkstra A. Understanding tailoring in communicating about health. Health Educ Res. 2008;23:454-66.

3. National Institute for Occupational Safety and Health. (2010). Protecting Yourself from Sun Exposure. Retrieved from: https:// www.cdc.gov/niosh/docs/2010-116/pdfs/2010-116.pdf

4. U.S. Preventive Services Task Force. (2018). Skin Cancer Prevention: Behavioral Counseling. Retrieved from: https://www. uspreventiveservicestaskforce.org/uspstf/recommendation/skincancer-counseling

5. Howie JG, Porter AM, Heaney DJ, Hopton JL. Long to short consultation ratio: a proxy measure of quality of care for general practice. Br J Gen Pract. 1991;41:48-54.

Copyright by Austin Hamp, et al. This is an open access article distributed under the terms of the Creative Commons Attribution License, which permits unrestricted use, distribution, and reproduction in any medium, provided the original author and source are credited.

Source of Support: Nil, Conflict of Interest: None declared. 